\section{Absolute proteome quantification in the gas-fermenting acetogen Clostridium}

\section{autoethanogenum}

\title{
ABSTRACT
}

Microbes that can recycle one-carbon (C1) greenhouse gases into fuels and chemicals are vital for the biosustainability of future industries. Acetogens are the most efficient known microbes for fixing carbon oxides $\mathrm{CO}_{2}$ and $\mathrm{CO}$. Understanding proteome allocation is important for metabolic engineering as it dictates metabolic fitness. Here, we use absolute proteomics to quantify intracellular concentrations for $>1,000$ proteins in the model-acetogen Clostridium autoethanogenum grown on three gas mixtures. We detect prioritisation of proteome allocation for $\mathrm{C} 1$ fixation and significant expression of proteins involved in the production of acetate and ethanol as well as proteins with unclear functions. The data also revealed which isoenzymes are important. Integration of proteomic and metabolic flux data demonstrated that enzymes catalyse high fluxes with high concentrations and high in vivo catalytic rates. We show that flux adjustments were dominantly accompanied with changing enzyme catalytic rates rather than concentrations. Our work serves as a reference dataset and advances systems-level understanding and engineering of acetogens. 


\section{INTRODUCTION}

30 Increasing concerns about irreversible climate change are accelerating the shift to renewable, carbon-

31 free energy production (e.g., solar, wind, fuel cells). However, many fuels and chemicals will stay

32 carbon-based, and thus, technologies for their production using sustainable and renewable feedstocks

33 are needed to transition towards a circular bioeconomy. Moreover, the rising amount of solid waste

34 produced by human activities (e.g., municipal solid waste, lignocellulosic waste) will further endanger our ecosystems' already critical state. Both challenges can be tackled by using organisms capable of recycling gaseous one-carbon $(\mathrm{C} 1)$ waste feedstocks (e.g., industrial waste gases $\left[\mathrm{CO}_{2}, \mathrm{CO}, \mathrm{CH}_{4}\right]$, syngas from gasified biomass or municipal solid waste $\left.\left[\mathrm{CO}, \mathrm{H}_{2}, \mathrm{CO}_{2}\right]\right)$ into fuels and chemicals at industrial scale $\mathrm{e}^{1-3}$.

As we transition into a new bioeconomy, a key feature of global biosustainability will be the capacity to convert carbon oxides into products at industrial scale. Acetogens are the ideal biocatalysts

41 for this as they use the most energy-efficient pathway, the Wood-Ljungdahl pathway (WLP) $)^{4,5}$, for

42 fixing $\mathrm{CO}_{2}$ into the central metabolite acetyl- $\mathrm{CoA}^{6-9}$ and accept gas $\left(\mathrm{CO}, \mathrm{H}_{2}, \mathrm{CO}_{2}\right)$ as their sole carbon

43 and energy source ${ }^{5}$. Indeed, the model-acetogen Clostridium autoethanogenum is already being used

44 as a cell factory in industrial-scale gas fermentation ${ }^{3,10}$. The WLP is considered the first biochemical

45 pathway on Earth ${ }^{7,11-13}$ and continues to play a critical role in the biogeochemical carbon cycle by

46 fixing an estimated $20 \%$ of the global $\mathrm{CO}_{2}{ }^{6,14}$. While biochemical details of the WLP are well

47 described $^{4,6,15}$, a quantitative understanding of acetogen metabolism is just emerging ${ }^{16,17}$. Notably,

48 recent systems-level analyses of acetogen metabolism have revealed mechanisms behind metabolic

49 shifts $^{18-21}$, transcriptional architectures ${ }^{22,23}$, and features of translational regulation ${ }^{24,25}$. However, we

50 still lack an understanding of acetogen proteome allocation through the quantification of proteome-

51 wide intracellular protein concentrations. This fundamental knowledge is required for advancing

52 rational metabolic engineering of acetogen cell factories and for accurate in silico reconstruction of

53 their phenotypes using metabolic models ${ }^{1,2}$.

Quantitative description of an organism's proteome allocation through absolute proteome

55 quantification is valuable in several ways. Firstly, it enables us to understand prioritisation of the

56 energetically costly proteome resources among functional protein categories, metabolic pathways, and 
57 single proteins ${ }^{26,27}$. This may also identify relevant proteins with unclear functions and high

58 abundances. Secondly, some metabolic fluxes can be catalysed by isoenzymes and a comparison of

59 their intracellular concentrations can indicate which are likely relevant in vivo and are thus targets for

60 genetic perturbation experiments to validate in vivo functionalities ${ }^{28}$. Thirdly, integration of absolute

61 proteomics and metabolic flux data enable the estimation of apparent in vivo catalytic rates of

62 enzymes $\left(\mathrm{k}_{\mathrm{app}}\right)^{26,29}$, which can be used to identify less-efficient enzymes as targets for improving

63 pathways through metabolic and protein engineering. Absolute proteomics data also contribute to the

64 curation of accurate genome-scale metabolic models.

65 Absolute proteome quantification is generally performed using label-free mass-spectrometry

66 (MS) approaches without spike-in standards $\mathrm{s}^{30,31}$. The major limitation of this approach is that

67 accuracy of label-free estimated protein concentrations cannot be determined. Furthermore, the

68 optimal model to convert MS signals (e.g., spectral counts, peak intensities) into protein

69 concentrations remains unknown ${ }^{32-34}$. Label-based approaches using stable-isotope labelled (SIL)

70 spike-ins of endogenous proteins are thus preferred for reliable absolute proteome quantification. This

71 strategy relies on accurate absolute quantification of a limited set of intracellular proteins (i.e.,

72 anchors) using SIL spike-ins to establish a linear correlation between protein concentrations and their

73 measured MS intensities ${ }^{32}$. Studies with the latter approach have determined a 1.5 $\square 2.4$-fold error for

74 label-free estimation of proteome-wide protein concentrations in multiple organisms ${ }^{28,35-41}$.

75 The aim of our work was to perform reliable absolute proteome quantification for the first

76 time in an acetogen. We employed a label-based MS approach using SIL-protein spike-in standards to

77 quantify SIL-based concentrations for 16 key proteins and label-free-based concentrations for >1,000

78 C. autoethanogenum proteins during autotrophic growth on three gas mixtures. This allowed us to

79 explore global proteome allocation, uncover isoenzyme usage in central metabolism, and quantify

80 regulatory principles associated with estimated $\mathrm{k}_{\mathrm{app}} \mathrm{s}$. Our work provides an important reference

81 dataset and advances the systems-level understanding and engineering of the ancient metabolism of

82 acetogens. 


\section{RESULTS}

85 Absolute proteome quantification framework in the model-acetogen C. autoethanogenum. We performed absolute proteome quantification from autotrophic steady-state chemostat cultures of $C$. autoethanogenum grown on three different gas mixtures: $\mathrm{CO}$, syngas $\left(\mathrm{CO}+\mathrm{CO}_{2}+\mathrm{H}_{2}\right)$, or $\mathrm{CO}+\mathrm{H}_{2}$ (termed "high- $\mathrm{H}_{2}$ CO") described before ${ }^{18,19}$. Briefly, four biological cultures of each gas mixture were grown anaerobically on a chemically defined medium at $37^{\circ} \mathrm{C}$, $\mathrm{pH}$ of 5 , and dilution rate $\sim 1$ day $^{-1}$ ( specific growth rate $\sim 0.04 \mathrm{~h}^{-1}$ ) without the use of heavy SIL substrates. The absolute proteome quantification framework (Fig. 1) was built on using 19 synthetic heavy SIL-variants of key $C$. autoethanogenum proteins covering central metabolism (Supplementary Table 1). The SIL-protein standards were spiked in for quantification of intracellular concentrations of their endogenous light counterparts. This framework ensures accurate absolute quantification compared to commonly used peptide spike-ins. Spiking cell lysates with protein standards before sample clean-up and protein digestion accounts for errors accompanying these critical steps ${ }^{30,31,42,43}$. Furthermore, selection of peptides ensuring accurate absolute protein quantification without prior MS data is challenging as its difficult to predict which peptides "fly" well ${ }^{30,31,42,43}$. In contrast, all proteotypic peptides from a protein spike-in can be used for quantification.

We synthesised heavy-labelled lysine and arginine SIL-proteins using a cell-free wheat germ extract platform as described previously ${ }^{18,44,45}$ and quantified standard stocks using parallel reaction monitoring (PRM) MS. Next, proteins were extracted from culture samples using an optimised protocol maximising extraction yield ${ }^{18}$ followed by spike-in of the 19 heavy SIL-proteins into light cell lysates. We then used a data-independent acquisition (DIA) MS approach ${ }^{46}$ to quantitate 1,243 proteins of $C$. autoethanogenum across 12 samples (quadruplicate cultures of three gas mixtures) using a comprehensive spectral library consisting of whole-cell lysates, lysate fractions, and spike-in SIL-proteins. Finally, we quantified intracellular concentrations for 16 key C. autoethanogenum proteins using light-to-heavy ratios between endogenous and spike-in DIA MS intensities and further used these 16 as anchor proteins for label-free estimation of $\sim 1,043$ protein concentrations through establishing a linear correlation between protein concentrations and their measured MS intensities ${ }^{32}$. 
111 We express intracellular protein concentrations in nanomoles of protein per gram of dry cell weight

$112(\mathrm{nmol} / \mathrm{gDCW})$.

114 Absolute quantification of 16 anchor protein concentrations. To ensure high confidence absolute quantification of anchor protein concentrations from the DIA MS data, we employed stringent criteria on top of the automated mProphet peak picking algorithm ${ }^{47}$ within the software Skyline ${ }^{48}$. We also performed a dilution series experiment for each SIL-protein to increase accuracy (see Methods for details). Briefly, we kept only peaks with Gaussian shapes and without interference and precursors with highest Skyline quality metrics. Importantly, only peptides whose signal were above the lower limit of quantification (LLOQ) and within the linear dynamic quantification range in the dilution series experiment were used for anchor protein quantification (Supplementary Table 2). We thus used 106 high-confidence peptides for the absolute quantification of 16 anchor protein concentrations (Table 1; see also Fig. 5). High confidence of the intracellular concentrations for these key $C$. autoethanogenum proteins of central metabolism is supported both by the low average $11 \%$ coefficient of variation (CV) between biological quadruplicate cultures (Table 1) and the average 22\% CV between different peptides of single proteins (Supplementary Table S2). and suitable anchor proteins are required for reliable label-free absolute proteome quantification. Our proteome-wide DIA MS data were highly reproducible with an average Pearson correlation coefficient of $\mathrm{R}=0.99$ between biological replicates (Fig. 2a and Supplementary Fig. 1). We also found our anchor proteins suitable as their concentrations spanned across three orders of magnitude, and the summed mass accounted for $\sim 1 / 3$ of the peptide mass injected into the mass spectrometer (Table 1 and Fig. 2b). We used the 16 anchor proteins (with 106 peptides) to determine the optimal label-free quantification model with the best linear fit between anchor protein concentrations and their measured DIA MS intensities using the aLFQ R package ${ }^{49}$ as described before for SWATH MS ${ }^{28}$ (Fig.

137 2c). Notably, we detected an average 1.5-fold cross-validated mean fold-error (CV-MFE;

138 bootstrapping) for the label-free estimated anchor protein concentrations across samples (Fig. 2d). 
139 The errors were distributed normally (Supplementary Fig. 2) with an average 95\% CI of 0.3 (Fig. 2d).

140 We then applied the optimal label-free quantification model to estimate $\sim 1,043$ protein concentrations

141 in C. autoethanogenum (Supplementary Table 3).

142 Prior to the detailed analysis of proteome-wide protein concentrations, we further evaluated

143 our label-free data accuracy beyond the 1.5-fold CV-MFE determined above. Firstly, the total

144 proteome mass $(1.2 \pm 0.1 \mu \mathrm{g}$; average \pm standard deviation) closely matched the $1 \mu \mathrm{g}$ peptide mass

145 injected into the mass spectrometer (Fig. 2d). The data were also supported by a strong correlation

146 between estimated protein concentrations and expected stoichiometries for equimolar (Fig. 3) and

147 non-equimolar protein complexes (Supplementary Fig. 3). Notably, absolute protein concentrations of

148 syngas cultures correlated well $(\mathrm{R}=0.65)$ with their respective absolute transcript expression levels

149 determined before ${ }^{19}$ (Supplementary Fig. 4). This result is similar to the correlations of absolute data

150 seen in other steady-state cultures ${ }^{26,50}$. Altogether, we present the first absolute quantitative proteome

151 dataset for a gas-fermenting acetogen that includes SIL-based concentrations for 16 key proteins and

152 label-free estimates for over 1,000 C. autoethanogenum proteins during growth on three gas mixtures.

154 C1 fixation dominates global proteome allocation. Global proteome allocation amongst functional

155 gene classifications was explored using proteomaps ${ }^{27}$ and KEGG Orthology identifiers (KO IDs) ${ }^{51}$.

156 The "treemap" structure defining the four-level hierarchy of our proteomaps (Supplementary Table 4)

157 also included manually curated categories to accurately reflect acetogen metabolism (e.g., C1

158 fixation/WLP, Hydrogenases). As expected for autotrophic growth of an acetogen, the C1 fixation

159 (Fig. 4) or WLP (Supplementary Fig. 5) categories dominated the proteome allocation with a $\sim 1 / 3$

160 fraction, compared to Carbohydrate metabolism or Glycolysis/Gluconeogenesis. Notably, the data

161 show that two genes-dihydrolipoamide dehydrogenase (LpdA; CAETHG_RS07825) and glycine

162 cleavage system H protein (GcvH; RS07795)-encoded by the WLP gene cluster were translated at

163 very high levels (Fig. 4). This is important as both have unknown functions in C. autoethanogenum

164 metabolism. Significant investment in expression of proteins involved in acetate and ethanol

165 production (Supplementary Fig. 5) is consistent with $1 / 3-$ to- $2 / 3$ of fixed carbon channelled into these

166 two growth by-products across the three gas mixtures ${ }^{18,19}$. The $11 \%$ proteome fraction of category 
167 Translation (Fig. 4) is expected for cells growing at a specific growth rate $\sim 0.04 \mathrm{~h}^{-1}$ based on absolute

168 proteomics data from Escherichia coli ${ }^{26,39,52}$. The notable proteome allocation for Amino acid

169 metabolism and particularly the high abundance of ketol-acid reductoisomerase (IlvC; RS00580) are

170 surprising since metabolic fluxes through 2,3-butanediol and branched-chain amino acid pathways

171 were low under these growth conditions ${ }^{18,19}$. In addition, numerous proteins with unknown or unclear

172 functions (coloured grey in proteomaps) are highly expressed (e.g., RS12590, RS08610, RS08145),

173 highlighting the need for global mapping of genotype-phenotype relationships in acetogens. In general,

174 proteome allocation was highly similar between the three gas mixtures (Supplementary Table 3). This

175 result is unsurprising given the few relative protein expression differences detected previously ${ }^{18}$.

177 Enzyme usage revealed in central metabolism. Next, we focused on uncovering enzyme usage in acetogen central metabolism (Fig. 5). This contains enzymes of the WLP, acetate, ethanol, and 2,3butanediol production pathways, hydrogenases, and the Nfn transhydrogenase, which together carry $>90 \%$ of the carbon and most of the redox flow in C. autoethanogenum ${ }^{18-20}$. Multiple metabolic fluxes in these pathways can be catalysed by isoenzymes and absolute proteomics data can indicate which of the isoenzymes are likely relevant in vivo. While the carbon monoxide dehydrogenase (CODH) AcsA (RS07861 $\square 62$ ) that forms the bifunctional CODH/ACS complex with the acetyl-CoA synthase ${ }^{53}$ (AcsB; RS07800) is essential for C. autoethanogenum growth on gas as confirmed in mutagenesis studies $^{54}$, the higher concentrations of the dispensable monofunctional CODH CooS1 (RS14775) suggest it may also play a role in $\mathrm{CO}$ oxidation (Fig. 4, 5), in addition to $\mathrm{CO}_{2}$ reduction ${ }^{54}$.

Additionally, our proteomics data show high abundance of the primary acetaldehyde:ferredoxin oxidoreductase (AOR1; RS00440) and this support the emerging understanding that in $C$. autoethanogenum ethanol is dominantly produced using the AOR1 activity via acetate, instead of directly from acetyl-CoA via acetaldehyde using mono- or bifunctional activities ${ }^{18,19,55,56}$ (Fig. 5). for reducing acetaldehyde to ethanol, a key reaction in terms of carbon and redox metabolism. The 193 high abundance of the electron-bifurcating hydrogenase HytA-E complex (RS13745 $\square 70$ ) compared 194 to alternative hydrogenases confirms that it is the main $\mathrm{H}_{2}$-oxidiser ${ }^{57,58}$ (Fig. 5). This is consistent with 
195 the fact that in the presence of $\mathrm{H}_{2}$ all the $\mathrm{CO}_{2}$ fixed by the WLP is reduced to formate using $\mathrm{H}_{2}$ by the

196 HytA-E and formate dehydrogenase (FdhA; RS13725) enzyme complex activity ${ }^{18,19}$. Despite the

197 proteomics evidence, genetic perturbations are required to determine condition-specific in vivo

198 functionalities of isoenzymes in acetogens unequivocally.

199 The overall most abundant protein was the formate-tetrahydrofolate ligase (Fhs; RS07850), a

200 key enzyme in the WLP (Fig. 5, 4). Despite the high abundance, its expression might still be rate-

201 limiting (see below). Another key enzyme for acetogens is AcsB because of its essentiality for acetyl-

202 CoA synthesis by the CODH/ACS complex. AcsB is linked to the WLP by the corrinoid iron sulfur

203 proteins AcsC (RS07810) and AcsD (RS07815) that supply the methyl group to AcsB. Interestingly,

204 the ratio of AcsCD-to-AcsB increased from 1.7 (CO) to 2.3 (syngas) to 2.9 (high- $\mathrm{H}_{2} \mathrm{CO}$ ), suggesting

205 that the primary role of the CODH/ACS complex shifted from CO oxidation towards acetyl-CoA

206 synthesis, likely because increased $\mathrm{H}_{2}$ uptake could replace the supply of reduced ferredoxin from $\mathrm{CO}$

207 oxidation. Concurrently, the Nfn transhydrogenase (RS07665) levels that act as a redox valve in

208 acetogens ${ }^{20}$ are maintained high (Fig. 5), potentially to rapidly respond to redox perturbations. We

209 conclude that absolute quantitative proteomics can significantly contribute to a systems-level

210 understanding of metabolism, particularly in less-studied organisms.

Integration of absolute proteomics and flux data yields in vivo enzyme catalytic rates. Absolute proteomics data enable estimation of intracellular catalytic working rates of enzymes when metabolic flux rates are known ${ }^{26,29}$. We thus calculated apparent in vivo catalytic rates of enzymes, denoted as $\mathrm{k}_{\mathrm{app}}\left(\mathrm{s}^{-1}\right)^{26}$, as the ratio of specific flux rate $(\mathrm{mmol} / \mathrm{gDCW} / \mathrm{h})$ determined before ${ }^{18}$ and protein concentration (nmol/gDCW) (see Methods). This produced $\mathrm{k}_{\mathrm{app}}$ values for 13 and 48 enzymes/complexes using either anchor or label-free protein concentrations, respectively (Supplementary Table 5 and Fig. 5, 6). The first two critical steps for carbon fixation in the methyl branch of the WLP (i.e., CO to formate) are catalysed at high rates (Fig. 5). Notably, FdhA showed a $\mathrm{k}_{\text {app }} \sim 30 \mathrm{~s}^{-1}$ for $\mathrm{CO}_{2}$ reduction without $\mathrm{H}_{2}$ during growth on $\mathrm{CO}$ only, which is similar to in vitro $\mathrm{k}_{\text {cat }}$ data of formate dehydrogenases in other acetogens ${ }^{59,60}$. Interestingly, the next step of formate reduction was catalysed potentially by a less-efficient enzyme-Fhs-as its $k_{\text {app }}$ of $\sim 3 \mathrm{~s}^{-1}$ is significantly 
223 lower compared to other WLP enzymes (Fig. 5). Overall, enzymes catalysing reactions in high flux

224 pathways such as the WLP and acetate and ethanol production have higher $\mathrm{k}_{\mathrm{app}} \mathrm{s}$ than those of

225 downstream from conversion of acetyl-CoA to pyruvate (Fig. 5). Indeed, enzymes catalysing high

226 metabolic fluxes in C. autoethanogenum have both higher concentrations and higher catalytic rates

227 compared to enzymes catalysing lower fluxes as both specific flux rates and enzyme concentrations

228 (Kendall's $\tau=0.56, \mathrm{p}$-value $\left.=5 \times 10^{-9}\right)$ and flux and $\mathrm{k}_{\text {app }}\left(\tau=0.45\right.$, $\mathrm{p}$-value $\left.=2 \times 10^{-6}\right)$ were significantly

229 correlated (Fig. 6a), as seen before for other organisms ${ }^{26,61}$.

230 Having acquired absolute proteomics data for C. autoethanogenum growth on three gas

231 mixtures with different metabolic flux profiles also allowed us to determine the impact of change in

232 enzyme concentration and its catalytic rate for adjusting metabolic flux rates. Two extreme examples

233 are the reactions catalysed by the HytA-E (Fig. 6b) and the Nfn (Fig. 5) complexes where flux

234 adjustments were accompanied with large changes in $\mathrm{k}_{\mathrm{app}} \mathrm{s}$ rather than in enzyme concentrations. Flux

235 changes in high flux pathways such as the WLP and acetate and ethanol production also coincided

236 mainly with $\mathrm{k}_{\text {app }}$ changes (Fig. 6b). This principle seems to be dominant in C. autoethanogenum as

$23790 \%$ of flux changes were not regulated through enzyme concentrations (i.e., post-translational

238 regulation; Supplementary Table 6) when comparing all statistically significant flux changes between

239 the three gas mixtures with respective enzyme expression changes (see Methods).

\section{DISCUSSION}

242 The looming danger of irreversible climate change and harmful effects of solid waste accumulation

243 are pushing humanity to develop and adopt sustainable technologies for renewable production of fuels

244 and chemicals and for waste recycling. Acetogen gas fermentation offers great potential to tackle both

245 challenges through recycling waste feedstocks (e.g., industrial waste gases, gasified biomass or

246 municipal solid waste) into fuels and chemicals ${ }^{1,2}$. Although the quantitative understanding of

247 acetogen metabolism has recently improved ${ }^{16,17}$, a quantitative description of acetogen proteome

248 allocation was missing. This is needed to advance their metabolic engineering into superior cell

249 factories and accurate in silico reconstruction of their phenotypes ${ }^{1,2}$. Thus, we performed absolute 
250 proteome quantification in the model-acetogen C. autoethanogenum grown autotrophically on three 251 gas mixtures.

Our absolute proteome quantification framework relied on SIL-protein spike-in standards and DIA MS analysis to ensure high confidence of the determined intracellular concentrations for 16 key estimation of $>1,000$ protein concentrations. This enabled us to determine the optimal label-free quantification model for our data to infer protein concentrations from MS intensities, which remains unknown in common label-free approaches not utilising spike-in standards ${ }^{32,33}$. More importantly, label-free estimated protein concentrations using the latter approach are questionable as their accuracy cannot be determined. We determined an excellent average error of 1.5-fold for our label-free estimated protein concentrations based on 16 anchor proteins and a bootstrapping approach. This error is in the same range as described in previous studies using SIL spike-in standards for absolute proteome quantification $^{28,35-41}$. Further, we also observed a good match both between estimated and injected proteome mass into the mass spectrometer and between protein concentrations and expected protein complex stoichiometries. We conclude that label-free estimation of proteome-wide protein concentrations using SIL-protein spike-ins and state-of-the-art MS analysis is reasonably accurate. prioritisation of proteome resources for fixing carbon through the WLP, in line with transcript expression data in C. autoethanogenum ${ }^{19,56}$. The allocation of one third of the total proteome for C1 fixation is higher than proteome allocation for carbon fixation through glycolysis during heterotrophic

270 growth of other microorganisms ${ }^{26,36}$. High abundances of other key enzymes of acetogen central metabolism were also expected as the WLP, acetate and ethanol production pathways, hydrogenases,

272 and the Nfn transhydrogenase carry $>90 \%$ of the carbon and most of the redox flow in $C$.

273 autoethanogenum ${ }^{18-20}$. However, very high expression of the two genes-LpdA and GcvH-of the WLP 274 gene cluster with unknown functions in C. autoethanogenum is striking, raising the question whether their function in C. autoethanogenum could also be to link WLP and glycine synthase-reductase pathways, as recently proposed for another acetogen ${ }^{62}$. Since many other proteins with unknown or 
277 unclear functions were also highly abundant, global mapping of genotype-phenotype relationships in 278 acetogens is much needed.

279 The in vivo functionalities of isoenzymes are not clear for multiple key metabolic fluxes in 280 acetogen central metabolism and absolute proteomics data can indicate which isoenzymes are likely relevant. Oxidation of $\mathrm{CO}$ or reduction of $\mathrm{CO}_{2}$ is a fundamental step for all acetogens and known to

282 be catalysed by three CODHs in C. autoethanogenum ${ }^{54}$. Though only AcsA that forms the

283 bifunctional CODH/ACS complex with the acetyl-CoA synthase ${ }^{53}$ is essential for growth on gas ${ }^{54}$, we

284 detected higher concentrations of the monofunctional CODH CooS1, which deletion strain shows

285 intriguing phenotypes ${ }^{54}$. Concurrently, our data suggest that prioritisation of CODH/ACS activity

286 between CO oxidation and acetyl-CoA synthesis is sensitive to $\mathrm{H}_{2}$ availability. Thus, further studies

287 are required to decipher condition-dependent functionalities of CODHs. In addition to CODHs, the

288 biochemical understanding of ethanol production is important in terms of both carbon and redox

289 metabolism. Our data confirm that in C. autoethanogenum ethanol is predominantly produced via

290 acetate by AOR $1^{18,19,55,56}$ and more importantly, indicate for the first time that AOR 1 activity is

291 followed by Adh4 (previously characterised as butanol dehydrogenase ${ }^{63}$ ) for reduction of

292 acetaldehyde to ethanol. These observations call for large-scale genetic perturbation experiments to

293 determine unequivocally the condition-specific in vivo functionalities of isoenzymes in acetogens.

294 Absolute proteomics data offer a unique opportunity to estimate apparent in vivo catalytic

295 rates of enzymes $\left(\mathrm{k}_{\mathrm{app}}\right)^{26,29}$ if also metabolic flux data are available. These data are particularly

296 valuable for more accurate in silico reconstruction of phenotypes using protein-constrained genome-

297 scale metabolic models ${ }^{64,65}$. While in vitro $\mathrm{k}_{\mathrm{cat}}$ and in vivo $\mathrm{k}_{\mathrm{app}}$ data generally correlate ${ }^{29}$, models using

298 maximal $k_{a p p}$ values show better prediction of protein abundances ${ }^{66}$. Furthermore, information of $k_{a p p} s$

299 can infer less-efficient enzymes as targets for improving pathways through metabolic and protein

300 engineering. For example, protein engineering of Fhs (catalysing formate reduction) might improve

301 WLP throughput and carbon fixation since its $\mathrm{k}_{\mathrm{app}}$ was significantly lower compared to other pathway

302 enzymes. At the same time, the large change in $\mathrm{k}_{\mathrm{app}} \mathrm{s}$ of the abundant electron-bifurcating hydrogenase

303 HytA-E and the Nfn transhydrogenase complexes indicate capacity for the cells to rapidly respond to

$304 \mathrm{H}_{2}$ availability and redox perturbations, which may be critical for metabolic robustness of acetogens ${ }^{20}$. 
305 Overall, we detected both higher concentrations and $\mathrm{k}_{\mathrm{app}} \mathrm{s}$ for enzymes catalysing higher metabolic

306 fluxes, which is believed to arise from an evolutionary push towards reducing protein production costs

307 for enzymes carrying high flux ${ }^{61}$. The observation that $90 \%$ of flux changes in C. autoethanogenum

308 were not regulated through changes in enzyme concentrations is not surprising for a metabolism that operates at the thermodynamic edge of feasibility ${ }^{16,17}$ since post-translational regulation of fluxes is energetically least costly. Further research is needed to identify which mechanism from posttranslational protein modification, allosteric regulation, or substrate concentration change is responsible for post-translational regulation of fluxes. understanding of global proteome allocation, isoenzyme usage in central metabolism, and regulatory principles of in vivo enzyme catalytic rates. This fundamental knowledge has potential to advance both rational metabolic engineering of acetogen cell factories and accurate in silico reconstruction of their phenotypes ${ }^{1,2,64,65}$. Our study also highlights the need for large-scale mapping of genotypephenotype relationships in acetogens to infer in vivo functionalities of isoenzymes and proteins with unknown or unclear functions. This absolute proteomics dataset serves as a reference towards a better systems-level understanding of the ancient metabolism of acetogens.

\section{METHODS}

323 Bacterial strain and culture growth conditions. Absolute proteome quantification was performed from high biomass concentration ( 1.4 gDCW/L) steady-state autotrophic chemostat cultures of $C$. autoethanogenum growing on three different gas mixtures with culturing conditions described in our previous works ${ }^{18,19}$. Briefly, four biological replicate chemostat cultures of C. autoethanogenum strain DSM 19630 were grown on a chemically defined medium (without yeast extract) either on CO ( 60\% $\mathrm{CO}$ and $40 \% \mathrm{Ar})$, syngas ( $~ 50 \% \mathrm{CO}, 20 \% \mathrm{H}_{2}, 20 \% \mathrm{CO}_{2}$, and $\left.10 \% \mathrm{~N}_{2} / \mathrm{Ar}\right)$, or $\mathrm{CO}+\mathrm{H}_{2}$, termed "highmaintained at $37^{\circ} \mathrm{C}, \mathrm{pH}$ of 5 , and dilution rate $\sim 1$ day $^{-1}$ ( specific growth rate $\sim 0.04 \mathrm{~h}^{-1}$ ). 
332 Cell-free synthesis of stable-isotope labelled protein standards. Twenty proteins covering $C$.

333 autoethanogenum central carbon metabolism, the HytA-E hydrogenase, and a ribosomal protein

334 (Supplementary Table 1) were selected for cell-free synthesis of SIL-proteins as described in ref. ${ }^{18}$.

335 Briefly, genes encoding for these proteins were synthesised by commercial gene synthesis services

336 (Biomatik). Target genes were sub-cloned into the cell-free expression vector pEUE01-His-N2

337 (CellFree Sciences) and transformed into Escherichia coli DH5 $\alpha$ from which plasmid DNA was

338 extracted and purified. Correct gene insertion into the pEUE01-His-N2 was verified by DNA

339 sequencing. Subsequently, cell-free synthesis of His-tag fused C. autoethanogenum proteins was

340 performed using the bilayer reaction method with the wheat germ extract WEPRO8240H (CellFree

341 Sciences) as described previously ${ }^{44,45}$. mRNAs for cell-free synthesis were prepared by an in vitro

342 transcription reaction while in vitro translation of target proteins was performed using a bilayer

343 reaction where the translation layer was supplemented with L-Arg- ${ }^{13} \mathrm{C}_{6},{ }^{15} \mathrm{~N}_{4}$ and L-Lys- ${ }^{13} \mathrm{C}_{6},{ }^{15} \mathrm{~N}_{2}$

344 (Wako) at final concentrations of $20 \mathrm{mM}$ to achieve high efficiency (>99 \%) for stable-isotope

345 labelling of proteins. The in vitro synthesised SIL-protein sequences also contained an N-terminal

346 amino acid sequence GYSFTTTAEK that was later used as a tag for quantification of the SIL-protein

347 stock concentration. Subsequently, SIL-proteins were purified using the Ni-Sepharose High-

348 Performance resin (GE Healthcare Life Sciences) and precipitated using methanol:chloroform:water

349 precipitation in Eppendorf Protein LoBind ${ }^{\circledR}$ tubes. Lastly, precipitated SIL-proteins were reconstituted

350 in $104 \mu \mathrm{L}$ of $8 \mathrm{M}$ urea ([UA]; Sigma-Aldrich) in $0.1 \mathrm{M}$ Trizma ${ }^{\circledR}$ base (pH 8.5) by vigorous vortexing

351 and stored at $-80{ }^{\circ} \mathrm{C}$ until further use.

353 Absolute quantification of SIL-protein standards using PRM MS. Concentrations of the twenty

354 synthesised SIL-protein standard stocks were determined using PRM MS preceded by in-solution

355 digestion of proteins and sample desalting and preparation for MS analysis.

\section{Sample preparation}

357 Only Eppendorf Protein LoBind ${ }^{\circledR}$ tubes and pipette tips were used for all sample preparation steps.

358 Firstly, $20 \mu \mathrm{L}$ of UA was added to $4 \mu \mathrm{L}$ of the SIL-protein standard stock used to determine the stock

359 concentration, and the mix was vortexed. Then, $1 \mu \mathrm{L}$ of $0.2 \mathrm{M}$ DTT (Promega) was added, followed 
360 by vortexing and incubation for $1 \mathrm{~h}$ at $37^{\circ} \mathrm{C}$ to reduce disulphide bonds. Sulfhydryl groups were

361 alkylated with $2 \mu \mathrm{L}$ of $0.5 \mathrm{M}$ iodoacetamide (IAA; Sigma-Aldrich), vigorous vortexing, and

362 incubation for $30 \mathrm{~min}$ at room temperature in the dark. Next, $75 \mu \mathrm{L}$ of $25 \mathrm{mM}$ ammonium bicarbonate

363 was added to dilute UA down to $2 \mathrm{M}$ concentration. Subsequently, 2 pmol ( $2 \mu \mathrm{L}$ of stock) of the nonlabelled AQUA ${ }^{\circledR}$ peptide HLEAAKGYSFTTTAEKAAELHK (Sigma-Aldrich) containing the quantification tag sequence GYSFTTTAEK was added to enable quantification of SIL-protein stock concentrations using MS analysis based on the ratio of heavy-to-light GYSFTTTAEK signals (see below). Protein digestion was performed for $16 \mathrm{~h}$ at $37{ }^{\circ} \mathrm{C}$ with $0.1 \mu \mathrm{g}$ of Trypsin/Lys-C mix $(1 \mu \mathrm{L}$ of stock; Promega) and stopped by lowering $\mathrm{pH}$ to 3 by the addition of $5 \mu \mathrm{L}$ of $10 \%$ (v/v) trifluoroacetic acid (TFA). wetted using $0.1 \%$ (v/v) formic acid (FA) in $100 \%$ acetonitrile (ACN), equilibrated with $0.1 \%$ FA in $70 \%(\mathrm{v} / \mathrm{v}) \mathrm{ACN}$, and washed with $0.1 \%$ FA before loading the sample and washing again with $0.1 \%$ FA. Peptides were eluted from the ZipTips with $0.1 \%$ FA in $70 \%$ ACN. Finally, samples were dried using a vacuum-centrifuge (Eppendorf) at $30{ }^{\circ} \mathrm{C}$ until dryness followed by reconstitution in $12 \mu \mathrm{L}$ of $0.1 \% \mathrm{FA}$ in $5 \% \mathrm{ACN}$ for subsequent MS analysis.

\section{LC method for PRM MS}

377 A Thermo Fisher Scientific UltiMate 3000 RSLCnano UHPLC system was used to elute the samples.

378 Each sample was initially injected $(6 \mu \mathrm{L})$ onto a Thermo Fisher Acclaim PepMap $\mathrm{C}_{18}$ trap reversedphase column ( $300 \mu \mathrm{m} \times 5 \mathrm{~mm}$ nano viper, $5 \mu \mathrm{m}$ particle size) at a flow rate of $15 \mu \mathrm{L} / \mathrm{min}$ using $2 \%$ $\mathrm{ACN}$ for $3 \mathrm{~min}$ with the solvent going to waste. The trap column was switched in-line with the separation column (GRACE Vydac Everest $C_{18}, 300 \AA 150 \mu \mathrm{m}$ x $150 \mathrm{~mm}, 2 \mu \mathrm{m}$ ) and the peptides were eluted using a flowrate of $3 \mu \mathrm{L} /$ min using $0.1 \%$ FA in water (buffer A) and $80 \% \mathrm{ACN}$ in buffer A (buffer B) as mobile phases for gradient elution. Following 3 min isocratic of $3 \%$ buffer B, peptide elution employed a 3-40\% ACN gradient for 28 min followed by $40-95 \%$ ACN for 1.5 min and 95\% equilibration step.

\section{PRM MS data acquisition}


388 The eluted peptides from the C18 column were introduced to the MS via a nano-ESI and analysed 389 using the Thermo Fisher Scientific Q-Exactive HF-X mass spectrometer. The electrospray voltage 390 was $1.8 \mathrm{kV}$ in positive ion mode, and the ion transfer tube temperature was $250{ }^{\circ} \mathrm{C}$. Full MS-scans 391 were acquired in the Orbitrap mass analyser over the range $\mathrm{m} / \mathrm{z} 550-560$ with a mass resolution of $30,000($ at $\mathrm{m} / \mathrm{z} 200)$. The AGC target value was set at $1.00 \mathrm{E}+06$ and maximum accumulation time 50 ms for full MS-scans. The PRM inclusion list included two mass values of 552.7640 and 556.7711. MS/MS spectra were acquired in the Orbitrap mass analyser with a mass resolution of $15,000(\mathrm{at} \mathrm{m} / \mathrm{z}$ 200). The AGC target value was set at $1.00 \mathrm{E}+06$ and maximum accumulation time $30 \mathrm{~ms}$ for MS/MS with an isolation window of $2 \mathrm{~m} / \mathrm{z}$. The loop count was set at 14 to gain greater MS/MS data. Raw

PRM MS data have been deposited to Panorama at

https://panoramaweb.org/Valgepea_Cauto_PRM.url (private reviewer account details: username:

panorama+reviewer27@proteinms.net; password: hSAwNUAL) with a ProteomeXchange

Consortium (http://proteomecentral.proteomexchange.org) dataset identifier PXD025760.

\section{PRM MS data analysis}

Analysis of PRM MS data was performed using the software Skyline ${ }^{48}$. The following parameters were used to extract PRM MS data for the quantification tag sequence GYSFTTTAEK: three precursor isotope peaks with a charge of $2(++)$ were included (monoisotopic; $\mathrm{M}+1$; $\mathrm{M}+2$ ); five of the most intense y product ions from ion 3 to last ion of charge state 1 and 2 among the precursor were picked; chromatograms were extracted with an ion match mass tolerance of $0.05 \mathrm{~m} / \mathrm{z}$ for product ions by including all matching scans; full trypsin specificity with two missed cleavages allowed for peptides with a length of 8-25 AAs; cysteine carbamidomethylation as a fixed peptide modification. ${ }^{13} \mathrm{C}(6){ }^{15} \mathrm{~N}(2) /+8.014 \mathrm{Da}(\mathrm{K})$ and ${ }^{13} \mathrm{C}(6){ }^{15} \mathrm{~N}(4) /+10.008 \mathrm{Da}(\mathrm{R})$, respectively. This translated into the SIL-proteins and the non-labelled AQUA ${ }^{\circledR}$ peptide possessing the tag GYSFTTTAEK with $\mathrm{m} / \mathrm{z}$ of 556.7711 and 552.7640, respectively. Hence, the concentrations of SIL-protein stocks were calculated based on the ratio of heavy-to-light GYSFTTTAEK signals and the spike-in of 2 pmol of the non- 
416 Skyline) and heavy and light peak areas $\left(\mathrm{R}^{2}>0.99\right.$; rdotp in Skyline) for all SIL-protein standard

417 stocks. No heavy GYSFTTTAEK signal was detected for protein CAETHG_RS16140 (an acetylating

418 acetaldehyde dehydrogenase in the NCBI annotation of sequence NC_022592.1 ${ }^{67}$ ), thus 19 SIL-

419 proteins could be used for following absolute proteome quantification in C. autoethanogenum

420 (Supplementary Table 1). PRM MS data with all Skyline processing settings can be viewed and

421 downloaded from Panorama at https://panoramaweb.org/Valgepea_Cauto_PRM.url (private reviewer

422 account details: username: panorama+reviewer27@ proteinms.net; password: hSAwNUAL).

424 Absolute proteome quantification in C. autoethanogenum using DIA MS. We used 19 synthetic

425 heavy SIL variants (see above) of key C. autoethanogenum proteins (Supplementary Table 1) as

426 spike-in standards for quantification of intracellular concentrations of their non-SIL counterparts

427 using a DIA MS approach ${ }^{46}$. Also, we performed a dilution series experiment for the spike-in SIL-

428 proteins to ensure accurate absolute quantification. We refer to these 19 intracellular proteins as

429 anchor proteins that were further used to estimate proteome-wide absolute protein concentrations in $C$.

430 autoethanogenum. This was achieved by determining the best linear fit between anchor protein

431 concentrations and their measured DIA MS intensities using the same strategy as described

432 previously $^{28}$.

\section{Preparation of spike-in SIL-protein standard mix and dilution series samples}

434 Only Eppendorf Protein LoBind ${ }^{\circledR}$ tubes and pipette tips were used for all preparation steps. The 19

435 spike-in SIL-protein standards that could be used for absolute proteome quantification in $C$.

436 autoethanogenum (see above) were mixed in two lots: 1) 'sample spike-in standard mix': SIL-protein

437 quantities matching estimated intracellular anchor protein quantities (i.e., expected light-to-heavy

$438[\mathrm{~L} / \mathrm{H}]$ ratios of $\sim 1$ ) based on label-free absolute quantification of the same samples in our previous

439 work $^{18}$; 2) 'dilution series standard mix': SIL-protein quantities doubling the estimated intracellular

440 anchor protein quantities for the dilution series sample with the highest SIL-protein concentrations.

441 To ensure accurate absolute quantification of anchor protein concentrations, a dilution series

442 experiment was performed to determine the linear dynamic quantification range and LLOQ for each

443 of the 19 spike-in SIL-proteins. Dilution series samples were prepared by making nine 2-fold dilutions 
444 of the 'dilution series spike-in standard mix' (i.e., 10 samples total for dilution series with a 512-fold

445 concentration span) in a constant $C$. autoethanogenum cell lysate background $(0.07 \mu \mathrm{g} / \mu \mathrm{L} ; 10$

$446 \mu \mathrm{g} /$ tube) serving as a blocking agent to avoid loss of purified SIL-proteins (to container and pipette tip

447 walls) and as a background proteome for accurate MS quantification of the linear range and LLOQ for

448 anchor proteins.

\section{$449 \quad$ Sample preparation}

450 C. autoethanogenum cultures were sampled for proteomics by immediate pelleting of $2 \mathrm{~mL}$ of culture

451 using centrifugation $\left(25,000 \times g\right.$ for $1 \mathrm{~min}$ at $\left.4{ }^{\circ} \mathrm{C}\right)$ and stored at $-80{ }^{\circ} \mathrm{C}$ until analysis. Details of

452 protein extraction and protein quantification in cell lysates are described previously ${ }^{18}$. In short, thawed

453 cell pellets were suspended in lysis buffer (containing SDS, DTT, and Trizma ${ }^{\circledR}$ base) and cell lysis

454 was performed using glass beads and repeating a 'lysis cycle' consisting of heating, bead beating,

455 centrifugation, and vortexing before protein quantification using the 2D Quant Kit (GE Healthcare

456 Life Sciences).

457 Sample preparation and protein digestion for MS analysis was based on the filter-aided

458 sample preparation (FASP) protocol $^{68}$. The following starting material was loaded onto an Amicon ${ }^{\circledR}$

459 Ultra-0.5 mL centrifugal filter unit (nominal molecular weight cut-off of 30,000; Merck Millipore): 1)

$46050 \mu \mathrm{g}$ of protein for one culture sample from each gas mixture (CO, syngas, or high- $\left.\mathrm{H}_{2} \mathrm{CO}\right)$ for

461 building the spectral library for DIA MS data analysis (samples 1-3); 2) $7 \mu$ g of protein for one

462 culture sample from either syngas or high- $\mathrm{H}_{2} \mathrm{CO}$ plus 'sample spike-in standard mix' for including

463 spike-in SIL-protein data to the spectral library (samples 4-5); 3) $15 \mu \mathrm{g}$ of protein for all 12 culture

464 samples (biological quadruplicates from $\mathrm{CO}$, syngas, and high- $\mathrm{H}_{2} \mathrm{CO}$ ) plus 'sample spike-in standard

465 mix' for performing absolute proteome quantification in C. autoethanogenum (samples 6-17); 4) ten

466 dilution series samples with 10-15 $\mu \mathrm{g}$ of total protein (C. autoethanogenum cell lysate background

467 plus 'dilution series spike-in standard mix') for performing the dilution series experiment for the 19

468 spike-in SIL-proteins (see above) (samples 18-27).

469 Samples containing SIL-proteins (samples 4-27) were incubated at $37^{\circ} \mathrm{C}$ for $1 \mathrm{~h}$ to reduce

470 SIL-protein disulphide bonds (cell lysate contained DTT). Details of the FASP workflow are

471 described before ${ }^{18}$. In short, samples were washed with UA, sulfhydryl groups alkylated with IAA, 
472 proteins digested using a Trypsin/Lys-C mix, and peptides eluted from the filter with $60 \mu \mathrm{L}$ of

473 ammonium bicarbonate. Next, $50 \mu \mathrm{L}$ of samples 1-3 were withdrawn and pooled for performing high

$474 \mathrm{pH}$ reverse-phase fractionation as described previously ${ }^{18}$ for expanding the spectral library for DIA

475 MS data analysis, yielding eight fractions (samples 28-35). Subsequently, all samples were vacuum-

476 centrifuged at $30{ }^{\circ} \mathrm{C}$ until dryness followed by reconstitution of samples $1-3$ and $4-35$ in 51 and $13 \mu \mathrm{L}$

477 of $0.1 \%$ FA in $5 \%$ ACN, respectively. Finally, total peptide concentration in each sample was

478 determined using the Pierce ${ }^{\mathrm{TM}}$ Quantitative Fluorometric Peptide Assay (Thermo Fisher Scientific) to

479 ensure that the same total peptide amount across samples 1-17 and 28-35 (excluding samples 18-27,

480 see below) could be injected for DIA MS analysis.

481 LC method for data-dependent acquisition (DDA) and DIA MS

482 Details of the LC method employed for generating the spectral library using DDA and for DIA sample 483 runs are described previously ${ }^{20}$. In short, a Thermo Fisher Scientific UHPLC system including $\mathrm{C}_{18}$

484 trap and separation columns was used to elute peptides with a gradient and total elution time of 110

485 min. For each DDA and DIA sample run, $1 \mu \mathrm{g}$ of peptide material from protein digestion was injected,

486 except for dilution series samples (samples 18-27 above) that were injected in a constant volume of 3

$487 \mu \mathrm{L}$ to maintain the dilution levels of the 'dilution series spike-in standard mix'.

\section{DDA MS spectral library generation}

489 The following 13 samples were analysed on the Q-Exactive HF-X in DDA mode to yield the spectral

490 library for DIA MS data analysis: 1) three replicates of one culture sample from each gas mixture (CO,

491 syngas, or high- $\mathrm{H}_{2} \mathrm{CO}$ ) (samples 1-3 above); 2) three replicates of one culture sample from either syngas or high- $\mathrm{H}_{2} \mathrm{CO}$ plus 'sample spike-in standard mix' (samples 4-5); 3) eight high pH reverse-

493 phase fractions of a pool of samples from each gas mixture (samples 28-35).

Details of DDA MS acquisition for generating the spectral library are described before ${ }^{20}$. In

495 short, eluted peptides from the $\mathrm{C}_{18}$ column were introduced to the MS via a nano-ESI and analysed

496 using the Q-Exactive HF-X with an Orbitrap mass analyser. The DDA MS spectral library for DIA

497 MS data confirmation and quantification using the software Skyline ${ }^{48}$ was created using the Proteome

498 Discoverer 2.2 software (Thermo Fisher Scientific) and its SEQUEST HT search as described

499 previously $^{18}$. The final .pd result file contained peptide-spectrum matches (PSMs) with q-values 
500 estimated at $1 \%$ false discovery rate (FDR) for peptides $\geq 4$ AAs. The generated spectral library file

501 has been deposited to the ProteomeXchange Consortium

502 (http://proteomecentral.proteomexchange.org) via the PRIDE partner repository ${ }^{69}$ with the dataset 503 identifier PXD025732 (private reviewer account details: username: reviewer_pxd025732@ebi.ac.uk; 504 password: QdkJHXy0).

\section{DIA MS data acquisition}

506 Details of DIA MS acquisition are described before ${ }^{20}$. In short, as for DDA MS acquisition, eluted 507 peptides were introduced to the MS via a nano-ESI and analysed using the Q-Exactive HF-X with an 508 Orbitrap mass analyser. DIA was achieved using an inclusion list: m/z $395 \square 1100$ in steps of 15 amu and scans cycled through the list of 48 isolation windows with a loop count of 48 . In total, DIA MS

510 data was acquired for 22 samples (samples 6-27 defined in section Sample preparation). Raw DIA

511 MS data have been deposited to the ProteomeXchange Consortium

512 (http://proteomecentral.proteomexchange.org) via the PRIDE partner repository ${ }^{69}$ with the dataset 513 identifier PXD025732 (private reviewer account details: username: reviewer_pxd025732@ebi.ac.uk; 514 password: QdkJHXy0).

\section{$515 \quad$ DIA MS data analysis}

516 DIA MS data analysis was performed with Skyline ${ }^{48}$ as described before ${ }^{18}$ with the following 517 modifications: 1) 12 manually picked high confidence endogenous peptides present in all samples and 518 spanning the elution gradient were used for iRT alignment through building an RT predictor; 2) outlier peptides from iRT regression were removed; 3) a minimum of three isotope peaks were required for a precursor; 4) single peptide per spike-in SIL-protein was allowed for anchor protein absolute quantification while at least two peptides per protein were required for label-free estimation of proteome-wide protein concentrations; 5) extracted ion chromatograms (XICs) were transformed using Savitszky-Golay smoothing. Briefly, the .pd result file from Proteome Discoverer was used to build the DIA MS spectral library and the mProphet peak picking algorithm ${ }^{47}$ within Skyline was used to separate true from false positive peak groups (per sample) and only peak groups with q-value $<0.01$

526 (representing 1\% FDR) were used for further quantification. We confidently quantitated 7,288

527 peptides and 1,243 proteins across all samples and 4,887 peptides and 1,043 proteins on average 
528 within each sample for estimating proteome-wide absolute protein concentrations. For absolute

529 quantification of anchor protein concentrations, we additionally manually: 1) removed integration of

530 peaks showing non-Gaussian shapes or interference from other peaks; 2) removed precursors with

531 similarity measures of $\mathrm{R}^{2}<0.9$ between product peak areas and corresponding intensities in the

532 spectral library (dotp in Skyline), precursor peak areas and expected isotope distribution (idotp), or

533 heavy and light peak areas (rdotp). After analysis in Skyline, 17 spike-in SIL-proteins remained for

534 further analysis as protein CAETHG_RS14410 was not identified in DIA MS data while

535 CAETHG_RS18395 did not pass quantification filters (Supplementary Table 1). DIA MS data with

536 all Skyline processing settings can be viewed and downloaded from Panorama at

537 https://panoramaweb.org/Valgepea_Cauto Anchors.url for anchor protein absolute quantification and

538 at https://panoramaweb.org/Valgepea_Cauto_LF.url for estimating proteome-wide absolute protein

539 concentrations (private reviewer account details: username: panorama+reviewer27@ proteinms.net;

540 password: hSAwNUAL).

541 Absolute quantification of anchor protein concentrations

542 We employed further stringent criteria on top of the output from Skyline analysis to ensure high

543 confidence absolute quantification of 17 anchor protein concentrations. Firstly, precursor with highest

544 heavy intensity for the highest 'dilution series spike-in standard mix' sample in the dilution series

545 (DS01) was kept while others were deleted. Peptides quantified in less than three biological replicates

546 cultures within a gas mixture, with no heavy signal for DS01 sample, or with heavy signals for less

547 than three continuous dilution series samples were removed. Next, we utilised the dilution series

548 experiment to only keep signals over the LLOQ and within the linear dynamic quantification range.

549 For this, correlation between experimental and expected peptide $\mathrm{L} / \mathrm{H}$ signal ratios for each peptide

550 across the dilution series was made to determine the LLOQ and calculate correlation, slope, and

551 intercept between MS signal and spike-in level (Supplementary Table 2). Only peptides showing

552 correlation $\mathrm{R}^{2}>0.95,0.95<$ slope $<1.05$, and $-0.1<$ intercept $<0.1$ for the dilution series were kept. This

553 ensured that we were only using peptides within the linear dynamic range. The remaining peptides

554 were further filtered for each culture sample by removing peptides whose light or heavy signal was

555 below the LLOQ in the dilution series. Subsequently, only peptides were kept with L/H ratios for at 
556 least three biological replicates cultures for each gas mixture (i.e., $\geq 9$ data points). Finally, we aimed

557 to detect outlier peptides by calculating the \% difference of a peptide's L/H ratio from the average

558 L/H ratio of all peptides for a given protein for every sample. Peptides were considered outliers and

559 thus removed if the average difference across all samples was $>50 \%$ or if the average difference

560 within biological replicate cultures was $>50 \%$. After the previous stringent criteria were applied, 106

561 high-confidence peptides remained (Supplementary Table 2) for the quantification of 16 anchor

562 protein concentrations since CAETHG_RS01830 was lost during manual analysis (Table 1 and

563 Supplementary Table 1). Proteins CAETHG_RS13725 and CAETHG_RS07840 were excluded from

564 the high- $\mathrm{H}_{2} \mathrm{CO}$ culture dataset as their calculated concentrations varied $>50 \%$ between biological

565 replicates. Data of one high- $\mathrm{H}_{2} \mathrm{CO}$ culture was excluded from further analysis due to difference from

566 bio-replicates likely due to challenges with MS analysis.

567 Label-free estimation of proteome-wide protein concentrations

568 We used the anchor proteins to estimate proteome-wide protein concentrations in $C$.

569 autoethanogenum by determining the best linear fit between anchor protein concentrations and their

570 measured DIA MS intensities using the aLFQ R package ${ }^{49}$ and the same strategy as described for

571 SWATH MS ${ }^{28}$. Briefly, aLFQ used anchor proteins and cross-validated model selection by

572 bootstrapping to determine the optimal model within various label-free absolute proteome

573 quantification approaches (e.g., TopN, iBAQ). The approach can obtain the model with the smallest

574 error between anchor protein concentrations determined using SIL-protein standards and label-free

575 estimated concentrations. The models with the highest accuracy were used to estimate proteome-wide

576 label-free concentrations for all proteins from their DIA MS intensities (1,043 proteins on average

577 within each sample; minimal two peptides per protein; see above): summing the five most intense

578 fragment ion intensities of the most or three of the most intense peptides per protein for $\mathrm{CO}$ or high-

$579 \mathrm{H}_{2} \mathrm{CO}$ cultures, respectively; summing the five most intense fragment ion intensities of all quantified

580 peptides of the protein divided by the number of theoretically observable peptides (i.e., $\mathrm{iBAQ}^{70}$ ) for

581 syngas cultures. 
583 Expected protein complex stoichiometries. Equimolar stoichiometries for the HytA-E/FdhA and

584 MetFV protein complexes were expected based on SDS gel staining experiments in $C$.

585 autoethanogenum $^{57}$ and the acetogen Moorella thermoacetica ${ }^{71}$, respectively. Expected

586 stoichiometries for other protein complexes in Fig. 3 and Supplementary Fig. 3 were based on

587 measured stoichiometries in E. coli K-12 (Complex Portal; www.ebi.ac.uk/complexportal) and

588 significant homology between complex member proteins in C. autoethanogenum and E. coli. All

589 depicted C. autoethanogenum protein complex members had NCBI protein-protein BLAST E-

590 values $<10^{-16}$ and scores $>73$ against respective $E$. coli $\mathrm{K}-12$ proteins using non-redundant protein

591 sequences.

592

593 Generation of proteomaps. The distribution of proteome-wide protein concentrations among

594 functional gene classifications was visualised using proteomaps ${ }^{27}$. For this, the NCBI annotation of

595 sequence NC_022592.1 ${ }^{67}$ was used as the annotation genome for C. autoethanogenum, with

596 CAETHG_RS07860 removed and replaced with the carbon monoxide dehydrogenase genes named

597 CAETHG_RS07861 and CAETHG_RS07862 with initial IDs of CAETHG_1620 and 1621,

598 respectively. Functional categories were assigned to protein sequences with KO IDs ${ }^{51}$ using the

599 KEGG annotation tool BlastKOALA ${ }^{72}$. Since proteomaps require a tree-like hierarchy, proteins that

600 were automatically assigned to multiple functional categories were manually assigned to one bottom-

601 level category (Level 3 in Supplementary Table 4) based on their principal task. We also created

602 functional categories "C1 fixation/Wood-Ljungdahl Pathway" (Level 2/3), "Acetate \& ethanol

603 synthesis" (Level 3), "Energy conservation" (Level 3), "Hydrogenases" (Level 3) and manually

604 assigned key acetogen proteins to these categories to reflect more accurately functional categories for

605 an acetogen. Proteins without designated KO IDs were manually assigned to latterly created

606 categories or grouped under "Not Included in Pathway or Brite" (Level 1) with Level 2 and 3 as "No

607 KO ID”. If BlastKOALA assigned multiple genes the same proposed gene/protein name, unique

608 numbers were added to names (e.g., pfkA, pfkA2). The final "treemap" defining the hierarchy for our

609 proteomaps is in Supplementary Table 4.

610 
611 Calculation of apparent in vivo catalytic rates of enzymes $\left(\mathbf{k}_{\mathbf{a p p}}\right)$. We calculated apparent in vivo

612 catalytic rates of enzymes, denoted as $\mathrm{k}_{\mathrm{app}}\left(\mathrm{s}^{-1}\right)^{26}$, as the ratio of specific flux rate $(\mathrm{mmol} / \mathrm{gDCW} / \mathrm{h})$

613 determined before ${ }^{18}$ and protein concentration $(\mathrm{nmol} / \mathrm{gDCW})$ quantified here for the same $C$.

614 autoethanogenum $\mathrm{CO}$, syngas, and high- $\mathrm{H}_{2} \mathrm{CO}$ cultures. Gene-protein-reaction (GPR) data of the

615 genome-scale metabolic model iCLAU786 ${ }^{18}$ were manually curated to reflect most recent knowledge

616 and were used to link metabolic fluxes with catalysing enzymes. For reactions with multiple assigned

617 enzymes (i.e,. isoenzymes), the enzyme with the highest average ranking of its concentration across

618 the three cultures (Supplementary Table 3) was assumed to solely catalyse the flux. For enzyme

619 complexes, average of quantified subunit concentrations was used (standard deviation estimated using

620 error propagation). For the HytA-E hydrogenase, its measured protein concentration was split

621 between reactions "rxn08518_c0" (direct $\mathrm{CO}_{2}$ reduction with $\mathrm{H}_{2}$ in complex with FdhA) and

622 "leq000001_c0" ( $\mathrm{H}_{2}$ oxidation solely by HytA-E) proportionally to flux for syngas and high- $\mathrm{H}_{2} \mathrm{CO}$

623 cultures. The resulting enzymes or enzyme complexes catalysing specific fluxes are shown in

624 Supplementary Table 5. Finally, we assumed each protein chain being catalytically active and only

625 calculated $\mathrm{k}_{\text {app }}$ values for metabolic reactions with a non-zero flux in at least one condition, specific

626 flux rate $>0.1 \%$ of $\mathrm{CO}$ fixation flux in at least one condition, and label-free data with measured

627 concentration for the associated enzyme(s) in all conditions (Supplementary Table 5). Membrane

628 proteins were excluded from $\mathrm{k}_{\text {app }}$ calculations to avoid bias from potentially incomplete protein

629 extraction. This produced $\mathrm{k}_{\text {app }}$ values for 13 enzymes/complexes using anchor protein concentrations

630 and for 48 enzymes/complexes using label-free protein concentrations (Supplementary Table 5).

632 Determination of regulation level of metabolic fluxes. We used published flux and relative

633 proteomics data ${ }^{18}$ of the same cultures studied here to determine whether fluxes are regulated by

634 changing enzyme concentrations or their catalytic rates by considering metabolic fluxes with non-zero

635 specific flux rates in at least two conditions of $\mathrm{CO}$, syngas, or high- $\mathrm{H}_{2} \mathrm{CO}$ cultures. The same

636 manually curated GPRs and criteria for isoenzymes and protein complexes as described above for $\mathrm{k}_{\text {app }}$

637 calculation were used to determine flux-enzyme pairs (Supplementary Table 6). We first used a two-

638 tailed two-sample equal variance Student's t-test with FDR correction ${ }^{73}$ to determine fluxes with 
639 significant changes between any two conditions (q-value<0.05). We then used the Student's left-tailed

640 t-distribution with FDR to determine if the significant flux change for every flux was significantly

641 different from the change in respective enzyme expression between the same conditions

642 (Supplementary Table 6). Flux with a q-value $<0.05$ for the latter test was considered to be regulated at 643 post-translational level (e.g., by changing enzyme catalytic rate).

\section{DATA AVAILABILITY}

646 All data generated or analysed during this study are in the main text, supplementary information files, 647 or public databases. Raw PRM MS data have been deposited to Panorama at https://panoramaweb.org/Valgepea_Cauto_PRM.url (private reviewer account details: username: panorama+reviewer27@ proteinms.net; password: hSAwNUAL) with a ProteomeXchange Consortium (http://proteomecentral.proteomexchange.org) dataset identifier PXD025760. Raw DIA MS data have been deposited to the ProteomeXchange Consortium (http://proteomecentral.proteomexchange.org) via the PRIDE partner repository ${ }^{69}$ with the dataset identifier PXD025732 (private reviewer account details: username: reviewer_pxd025732@ebi.ac.uk; password: QdkJHXy0). PRM MS data with all Skyline processing settings can be viewed and downloaded from Panorama at https://panoramaweb.org/Valgepea_Cauto_PRM.url. DIA MS data with all Skyline processing settings can be viewed and downloaded from Panorama at at https://panoramaweb.org/Valgepea_Cauto_LF.url for estimating proteome-wide absolute protein concentrations (private reviewer account details: username: panorama+reviewer27@proteinms.net; password: hSAwNUAL). Any other relevant data are available from the corresponding author on reasonable request.

\section{REFERENCES}

664 1. Liew, F. et al. Gas Fermentation-A flexible platform for commercial scale production of low665 carbon-fuels and chemicals from waste and renewable feedstocks. Front. Microbiol. 7, 694 (2016). 
666 2. Claassens, N. J., Sousa, D. Z., dos Santos, V. A. P. M., de Vos, W. M. \& van der Oost, J.

667 Harnessing the power of microbial autotrophy. Nat. Rev. Microbiol. 14, 692-706 (2016).

668 3. Fackler, N. et al. Stepping on the gas to a circular economy: accelerating development of carbon-

669 negative chemical production from gas fermentation. Annu. Rev. Chem. Biomol. Eng. 12, 1 (2021).

670 4. Ragsdale, S. W. \& Pierce, E. Acetogenesis and the Wood-Ljungdahl pathway of $\mathrm{CO}_{2}$ fixation.

671 Biochim. Biophys. Acta 1784, 1873-1898 (2008).

672 5. Wood, H. G. Life with $\mathrm{CO}$ or $\mathrm{CO}_{2}$ and $\mathrm{H}_{2}$ as a source of carbon and energy. FASEB J. 5, 156-163

$673(1991)$

674 6. Drake, H. L., Küsel, K. \& Matthies, C. In The Prokaryotes Ch. Acetogenic Prokaryotes. 354-420

675 (2006).

676 7. Fuchs, G. Alternative pathways of carbon dioxide fixation: insights into the early evolution of life?

677 Ann. Rev. Microbiol. 65, 631-658 (2011).

678 8. Fast, A. G. \& Papoutsakis, E. T. Stoichiometric and energetic analyses of non-photosynthetic $\mathrm{CO}_{2^{-}}$

679 fixation pathways to support synthetic biology strategies for production of fuels and chemicals. Curr.

680 Opin. Chem. Eng. 1, 380-395 (2012).

681 9. Cotton, C. A., Edlich-Muth, C. \& Bar-Even, A. Reinforcing carbon fixation: $\mathrm{CO}_{2}$ reduction

682 replacing and supporting carboxylation. Curr. Opin. Biotechnol. 49, 49-56 (2018).

683 10. Köpke, M. \& Simpson, S. D. Pollution to products: recycling of 'above ground' carbon by gas

684 fermentation. Curr. Opin. Biotechnol. 65, 180-189 (2020).

685 11. Russell, M. J. \& Martin, W. The rocky roots of the acetyl-CoA pathway. Trends Biochem. Sci. 29, $686 \quad 358-363$ (2004).

687 12. Weiss, M. C. et al. The physiology and habitat of the last universal common ancestor. Nat.

688 Microbiol. 1, 16116 (2016).

689 13. Varma, S. J., Muchowska, K. B., Chatelain, P. \& Moran, J. Native iron reduces $\mathrm{CO}_{2}$ to

690 intermediates and end-products of the acetyl-CoA pathway. Nat. Ecol. Evol. 2, 1019-1024 (2018).

691 14. Ljungdahl, L. G. A life with acetogens, thermophiles, and cellulolytic anaerobes. Annu. Rev.

692 Microbiol. 63, 1-25 (2009). 
693 15. Ragsdale, S. W. Enzymology of the Wood-Ljungdahl pathway of acetogenesis. Ann. N. Y. Acad.

694 Sci. 1125, 129-136 (2008).

695 16. Schuchmann, K. \& Müller, V. Autotrophy at the thermodynamic limit of life: a model for energy

696 conservation in acetogenic bacteria. Nat. Rev. Microbiol. 12, 809-821 (2014).

697 17. Molitor, B., Marcellin, E. \& Angenent, L. T. Overcoming the energetic limitations of syngas

698 fermentation. Curr. Opin. Chem. Biol. 41, 84-92 (2017).

699 18. Valgepea, K. et al. $\mathrm{H}_{2}$ drives metabolic rearrangements in gas-fermenting Clostridium

700 autoethanogenum. Biotechnol. Biofuels 11, 55 (2018).

701 19. Valgepea, K. et al. Maintenance of ATP homeostasis triggers metabolic shifts in gas-fermenting

702 acetogens. Cell Syst. 4, 505-515.e5 (2017).

703 20. Mahamkali, V. et al. Redox controls metabolic robustness in the gas-fermenting acetogen

704 Clostridium autoethanogenum. Proc. Natl. Acad. Sci. U. S. A. 117, 13168-13175 (2020).

705 21. Richter, H. et al. Ethanol production in syngas-fermenting Clostridium ljungdahlii is controlled by

706 thermodynamics rather than by enzyme expression. Energy Environ. Sci. 9, 2392-2399 (2016).

707 22. de Souza Pinto Lemgruber, R. et al. A TetR-family protein (CAETHG_0459) activates

708 transcription from a new promoter motif associated with essential genes for autotrophic growth in 709 acetogens. Front. Microbiol. 10, 2549 (2019).

710 23. Song, Y. et al. Determination of the genome and primary transcriptome of syngas fermenting

711 Eubacterium limosum ATCC 8486. Sci. Rep. 7, 13694 (2017).

712 24. Al-Bassam, M. M. et al. Optimisation of carbon and energy utilisation through differential

713 translational efficiency. Nat. Commun. 9, 4474 (2018).

714 25. Song, Y. et al. Genome-scale analysis of syngas fermenting acetogenic bacteria reveals the

715 translational regulation for its autotrophic growth. BMC Genomics 19, 837 (2018).

716 26. Valgepea, K., Adamberg, K., Seiman, A. \& Vilu, R. Escherichia coli achieves faster growth by

717 increasing catalytic and translation rates of proteins. Mol. Biosyst. 9, 2344-2358 (2013).

718 27. Liebermeister, W. et al. Visual account of protein investment in cellular functions. Proc. Natl.

719 Acad. Sci. U. S. A. 111, 8488-8493 (2014). 
720 28. Schubert, O. T. et al. Absolute proteome composition and dynamics during dormancy and

721 resuscitation of Mycobacterium tuberculosis. Cell Host Microbe 18, 96-108 (2015).

722 29. Davidi, D. et al. Global characterisation of in vivo enzyme catalytic rates and their correspondence

723 to in vitro kcat measurements. Proc. Natl. Acad. Sci. U. S. A. 104, 20167-20172 (2016).

724 30. Calderón-Celis, F., Encinar, J. R. \& Sanz-Medel, A. Standardization approaches in absolute

725 quantitative proteomics with mass spectrometry. Mass Spectrom. Rev. 37, 715-737 (2017).

726 31. Maaß, S. \& Becher, D. Methods and applications of absolute protein quantification in microbial

727 systems. J. Proteomics 136, 222-233 (2016).

728 32. Ludwig, C., Claassen, M., Schmidt, A. \& Aebersold, R. Estimation of absolute protein quantities

729 of unlabeled samples by selected reaction monitoring mass spectrometry. Mol. Cell. Proteomics 11,

730 M111.013987 (2012).

731 33. Blein-Nicolas, M. \& Zivy, M. Thousand and one ways to quantify and compare protein

732 abundances in label-free bottom-up proteomics. Biochim. Biophys. Acta 1864, 883-895 (2016).

733 34. Ahrné, E., Molzahn, L., Glatter, T. \& Schmidt, A. Critical assessment of proteome-wide label-free

734 absolute abundance estimation strategies. Proteomics 17, 2567-2578 (2013).

735 35. Schmidt, A. et al. Absolute quantification of microbial proteomes at different states by directed

736 mass spectrometry. Mol. Syst. Biol. 7, 510 (2011).

737 36. Maier, T. et al. Quantification of mRNA and protein and integration with protein turnover in a

738 bacterium. Mol. Syst. Biol. 7, 511 (2011).

739 37. Marguerat, S. et al. Quantitative analysis of fission yeast transcriptomes and proteomes in

740 proliferating and quiescent cells. Cell 151, 671-683 (2012).

741 38. Zeiler, M., Moser, M. \& Mann, M. Copy number analysis of the murine platelet proteome

742 spanning the complete abundance range. Mol. Cell. Proteomics 13, 3435-3445 (2014).

743 39. Schmidt, A. et al. The quantitative and condition-dependent Escherichia coli proteome. Nat.

744 Biotechnol. 34, 104-110 (2015).

745 40. Beck, M. et al. The quantitative proteome of a human cell line. Mol. Syst. Biol. 7, 549 (2011).

746 41. Malmström, J. et al. Proteome-wide cellular protein concentrations of the human pathogen

747 Leptospira interrogans. Nature 460, 762-765 (2009). 
748 42. Brun, V. et al. Isotope-labeled protein standards: toward absolute quantitative proteomics. Mol.

749 Cell. Proteomics 6, 2139-2149 (2007).

750 43. Shuford, C. M. et al. Absolute protein quantification by mass spectrometry: not as simple as

751 advertised. Anal. Chem. 89, 7406-7415 (2017).

752 44. Takemori, N. et al. High-throughput synthesis of stable isotope-labeled transmembrane proteins

753 for targeted transmembrane proteomics using a wheat germ cell-free protein synthesis system. Mol.

754 Biosyst. 11, 361-365 (2015).

755 45. Takemori, N. et al. MEERCAT: multiplexed efficient cell free expression of recombinant

756 QconCATs for large scale absolute proteome quantification. Mol. Cell. Proteomics 16, 2169-2183

757 (2017).

758 46. Gillet, L. C. et al. Targeted data extraction of the MS/MS spectra generated by data-independent 759 acquisition: a new concept for consistent and accurate proteome analysis. Mol. Cell. Proteomics 11, $760 \quad 0111.016717(2012)$.

761 47. Reiter, L. et al. mProphet: automated data processing and statistical validation for large-scale 762 SRM experiments. Nat. Methods 8, 430-435 (2011).

763 48. MacLean, B. et al. Skyline: an open source document editor for creating and analysing targeted 764 proteomics experiments. Bioinformatics 26, 966-968 (2010).

765 49. Rosenberger, G., Ludwig, C., Röst, H. L., Aebersold, R. \& Malmström, L. aLFQ: an R-package 766 for estimating absolute protein quantities from label-free LC-MS/MS proteomics data. Bioinformatics 767 30, 2511-2513 (2014).

768 50. Lahtvee, P.-J. et al. Absolute quantification of protein and mRNA abundances demonstrate 769 variability in gene-specific translation efficiency in yeast. Cell Syst. 4, 495-504.e5 (2017).

770 51. Kanehisa, M., Sato, Y., Kawashima, M., Furumichi, M. \& Tanabe, M. KEGG as a reference

771 resource for gene and protein annotation. Nucleic Acids Res. 44, D457-D462 (2016).

772 52. Peebo, K. et al. Proteome reallocation in Escherichia coli with increasing specific growth rate.

773 Mol. Biosyst. 11, 1184-1193 (2015). 
774 53. Lemaire, O. N. \& Wagner, T. Gas channel rerouting in a primordial enzyme: Structural insights of

775 the carbon-monoxide dehydrogenase/acetyl-CoA synthase complex from the acetogen Clostridium

776 autoethanogenum. Biochim. Biophys. Acta 1862, 148330 (2021).

777 54. Liew, F. et al. Insights into $\mathrm{CO}_{2}$ fixation pathway of Clostridium autoethanogenum by targeted

778 mutagenesis. mBio 7, e00427-16 (2016).

779 55. Liew, F. et al. Metabolic engineering of Clostridium autoethanogenum for selective alcohol

780 production. Metab. Eng. 40, 104-114 (2017).

781 56. Marcellin, E. et al. Low carbon fuels and commodity chemicals from waste gases - systematic

782 approach to understand energy metabolism in a model acetogen. Green Chem. 18, 3020-3028 (2016).

783 57. Wang, S. et al. NADP-specific electron-bifurcating [FeFe]-hydrogenase in a functional complex

784 with formate dehydrogenase in Clostridium autoethanogenum grown on CO. J. Bacteriol. 195, 4373-

$7854386(2013)$.

786 58. Mock, J. et al. Energy conservation associated with ethanol formation from $\mathrm{H}_{2}$ and $\mathrm{CO}_{2}$ in

787 Clostridium autoethanogenum involving electron bifurcation. J. Bacteriol. 197, 2965-2980 (2015).

788 59. Maia, L. B., Fonseca, L., Moura, I. \& Moura, J. J. G. Reduction of carbon dioxide by a

789 molybdenum-containing formate dehydrogenase: a kinetic and mechanistic study. J. Am. Chem. Soc.

790 138, 8834-8846 (2016).

791 60. Schuchmann, K. \& Müller, V. Direct and reversible hydrogenation of $\mathrm{CO}_{2}$ to formate by a

792 bacterial carbon dioxide reductase. Science 342, 1382-1385 (2013).

793 61. Bar-Even, A. et al. The moderately efficient enzyme: evolutionary and physicochemical trends

794 shaping enzyme parameters. Biochemistry 50, 4402-4410 (2011).

795 62. Song, Y. et al. Functional cooperation of the glycine synthase-reductase and Wood-Ljungdahl

796 pathways for autotrophic growth of Clostridium drakei. Proc. Natl. Acad. Sci. U. S. A. 117, 7516-

$7977523(2020)$.

798 63. Tan, Y., Liu, J., Liu, Z. \& Li, F. Characterization of two novel butanol dehydrogenases involved

799 in butanol degradation in syngas-utilising bacterium Clostridium ljungdahlii DSM 13528. J. Basic

800 Microbiol. 54, 996-1004 (2014). 
801 64. Davidi, D. \& Milo, R. Lessons on enzyme kinetics from quantitative proteomics. Curr. Opin.

802 Biotechnol. 46, 81-89 (2017).

803 65. Nilsson, A., Nielsen, J. \& Palsson, B. Ø. Metabolic models of protein allocation call for the 804 kinetome. Cell Syst. 5, 538-541 (2017).

66. Heckmann, D. et al. Machine learning applied to enzyme turnover numbers reveals protein

806 structural correlates and improves metabolic models. Nat. Commun. 9, 5252 (2018).

807 67. Brown, S. D. et al. Comparison of single-molecule sequencing and hybrid approaches for

808 finishing the genome of Clostridium autoethanogenum and analysis of CRISPR systems in industrial

809 relevant Clostridia. Biotechnol. Biofuels 7, 40 (2014).

810 68. Wiśniewski, J. R., Zougman, A., Nagaraj, N. \& Mann, M. Universal sample preparation method

811 for proteome analysis. Nat. Methods 6, 359-362 (2009).

812 69. Vizcaíno, J. A. et al. The Proteomics Identifications (PRIDE) database and associated tools: status

813 in 2013. Nucleic Acids Res. 41, D1063-D1069 (2012).

814 70. Schwanhäusser, B. et al. Global quantification of mammalian gene expression control. Nature 473, $815 \quad 337-342(2011)$.

816 71. Mock, J., Wang, S., Huang, H., Kahnt, J. \& Thauer, R. K. Evidence for a hexaheteromeric 817 methylenetetrahydrofolate reductase in Moorella thermoacetica. J. Bacteriol. 196, 3303-3314 (2014).

818 72. Kanehisa, M., Sato, Y. \& Morishima, K. BlastKOALA and GhostKOALA: KEGG tools for

819 functional characterisation of genome and metagenome sequences. J. Mol. Biol. 428, 726-731 (2016).

820 73. Benjamini, Y. \& Hochberg, Y. Controlling the false discovery rate: a practical and powerful

821 approach to multiple testing. J. R. Stat. Soc. B 57, 289-300 (1995).

\section{ACKNOWLEDGEMENTS}

824 We thank Jörg Bernhardt for help with proteomaps, Tim McCubbin for scripts, Andrus Seiman for 825 statistics, and Olivier Lemaire for valuable discussions. This work was funded by the Australian 826 Research Council (ARC LP140100213) in collaboration with LanzaTech. We thank the following 827 investors in LanzaTech's technology: Sir Stephen Tindall, Khosla Ventures, Qiming Venture Partners, 828 Softbank China, the Malaysian Life Sciences Capital Fund, Mitsui, Primetals, CICC Growth Capital 
829 Fund I, L.P. and the New Zealand Superannuation Fund. The research utilised equipment and support

830 provided by the Queensland node of Metabolomics Australia, an initiative of the Australian

831 Government being conducted as part of the NCRIS National Research Infrastructure for Australia.

832 There was no funding support from the European Union for the experimental part of the study.

833 However, K.V. acknowledges support also from the European Union's Horizon 2020 research and

834 innovation programme under grant agreement N810755.

\section{AUTHOR CONTRIBUTIONS}

837 Conceptualisation, K.V., C.L., M.K., L.K.N., and E.M.; Methodology, K.V., G.T., N.T., A.T., C.L.,

838 A.P.M., R.T., and E.M.; Formal Analysis, K.V., and G.T.; Investigation, K.V., G.T., N.T., and A.T.;

839 Resources, N.T., A.T., M.K., S.D.S., L.K.N., and E.M.; Writing - Original Draft, K.V. and E.M.;

840 Writing - Review \& Editing, K.V., N.T., C.L., A.P.M., R.T., M.K., L.K.N., and E.M.; Supervision,

841 M.K., L.K.N., and E.M.; Project Administration, R.T., and E.M.; Funding Acquisition, M.K., S.D.S.,

842 L.K.N., and E.M..

\section{COMPETING INTERESTS}

LanzaTech has interest in commercial gas fermentation with C. autoethanogenum. A.P.M, R.T., M.K., and S.D.S. are employees of LanzaTech.

MATERIALS \& CORRESPONDENCE

849 Correspondence and request for materials should be addressed to E.M.

\section{FIGURE LEGENDS}

Fig. 1 Absolute proteome quantification framework in C. autoethanogenum. Absolute proteome quantification in light (no stable-isotope labelled [SIL] substrates) autotrophic C. autoethanogenum chemostat cultures was built on using 19 synthetic heavy SIL-protein spike-in standards and dataindependent acquisition (DIA) mass spectrometry (MS) analysis. Culture samples with SIL-protein 
857 analysis allowed to quantify intracellular concentrations for 16 key C. autoethanogenum proteins

858 using light-to-heavy ratios between endogenous and spike-in DIA MS intensities. These 16 key

859 proteins were further used as anchor proteins for label-free estimation of $\sim 1,043$ protein

860 concentrations through establishing a linear correlation between protein concentrations and their measured MS intensities. Some parts created with BioRender.com.

Fig. 2 Label-free estimation of proteome-wide protein concentrations. a Correlation of peptide mass spectrometry (MS) feature intensities between biological replicate cultures of the three gas mixtures. b Linear correlation between anchor protein concentrations and their measured MS intensities for one syngas culture. $g D C W$, gram of dry cell weight, $a L F Q$, absolute label-free quantification. c Errors of different label-free quantification models for the linear fit between anchor protein concentrations and their measured MS intensities determined by bootstrapping using the aLFQ $\mathrm{R}_{\text {package }}{ }^{49}$ for one syngas culture. $C V-M F E$, cross-validated mean fold-error. d Label-free quantification error of optimal model (orange) and total proteome mass (blue) across samples. Error bars denote $95 \%$ CI. equimolar protein complexes. Grey dotted lines denote the average 1.5 -fold cross-validated mean fold-error (CV-MFE) of label-free protein concentrations. Label-free protein concentrations are plotted, except for the HytA-FdhA complex, which was quantified using stable-isotope labelled protein spike-ins. Data points of the same colour represent gas mixtures. See Methods for details on expected protein complex stoichiometries. See Supplementary Table S3 for gene/protein ID, proposed name, description, and label-free data. See Table 1 for HytA-FdhA data. $g D C W$, gram of dry cell weight.

Fig. 4 Proteomaps uncover global proteome allocation. Left proteomap shows proteome allocation amongst functional gene classification categories (KEGG Orthology identifiers [KO IDs] ${ }^{51}$ ) at level two of the four-level "treemap" hierarchy structure (Supplementary Table S4). Right proteomap 
885 shows proteome allocation at the level of single proteins (level four of "treemap"). See Supplementary

886 Fig. S5 for proteomaps of levels one and three of "treemap". Area of the tile is proportional to protein

887 concentration. Colours denote level one categories of "treemap". Proteomaps visualise average

888 concentrations of syngas cultures while category percentages are average of three gas mixtures

889 (shown for categories with a fraction >5\%). See Supplementary Table S3 for gene/protein ID,

890 proposed name, description, and label-free protein concentrations.

Fig. 5 Quantitative systems-level view of acetogen central metabolism. Enzyme concentrations

$(\mathrm{nmol} / \mathrm{gDCW})$, apparent in vivo catalytic rates of enzymes $\left(\mathrm{k}_{\mathrm{app}} ; \mathrm{s}^{-1}\right)$, and metabolic flux rates

(mmol/gDCW/h) are shown for C. autoethanogenum steady-state chemostat cultures grown on three

gas mixtures. See dashed inset for bar chart and heatmap details. Enzyme concentration and $\mathrm{k}_{\text {app }}$ data

are average of biological replicates. Proteins forming a complex are highlighted with non-black heatmap), see Methods for details. Flux data from ref. ${ }^{18}$ are average of biological replicates and error bars denote standard deviation. Arrows show direction of calculated fluxes; red arrow denotes uptake or secretion. Gene/protein IDs right of enzyme concentration heatmaps are preceded with

CAETHG_RS and red font denotes concentrations determined using stable-isotope labelled (SIL) protein spike-in standards (i.e., anchor proteins). Asterisk denotes data for redox-consuming $\mathrm{CO}_{2}$ reduction to formate solely by FdhA without the use of $\mathrm{H}_{2}$ during growth on $\mathrm{CO}$. ${ }^{\mathrm{a} B i f u n c t i o n a l}$ acetaldehyde/alcohol dehydrogenase (acetyl-CoA $\rightarrow$ ethanol); ${ }^{b}$ Flux into PEP from OAA and pyruvate is merged and $\mathrm{k}_{\mathrm{app}}$ is for PEPCK. See Supplementary Table S3 for gene/protein ID, proposed name, description, and label-free protein concentrations. See Table 1 for anchor protein concentrations. See 
912 Fig. 6 Regulatory principles of apparent in vivo catalytic rates of enzymes $\left(\mathbf{k}_{\text {app }}\right)$ and metabolic

913 flux throughput. a Enzymes catalysing higher metabolic flux rates have both higher concentrations

914 and higher $k_{\text {app }} \mathrm{s}$. Yellow and blue denote high and low values, respectively. Kendall's $\tau$ correlations

915 with significance p-values between respective pairs are shown below heatmap. See Supplementary

916 Table S5 for flux rate, enzyme concentration, and $\mathrm{k}_{\text {app }}$ data, and for description of reaction names

917 (Rxn name) and gene-protein-reaction (GPR) associations. b Control of metabolic flux throughput

918 through $\mathrm{k}_{\text {app }}$ changes for high flux pathways. See also Fig. 5. $g D C W$, gram of dry cell weight. 
$\mathrm{CO} \mathrm{H}_{2} \mathrm{CO}_{2} \quad 19$ heavy SIL-proteins of $\quad$ Light and heavy

C. autoethanogenum

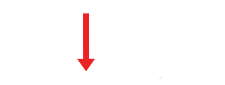



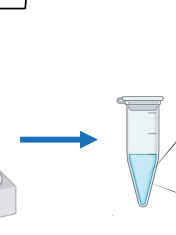

Light C.autoethanogenum chemostat cultures

Light cell lysate Light cell lysates \& fractions
DIA-MS

$$
\text { peptides Thermo Q-Exactive HF-X }
$$

Data analysis

Skyline \& mProphet

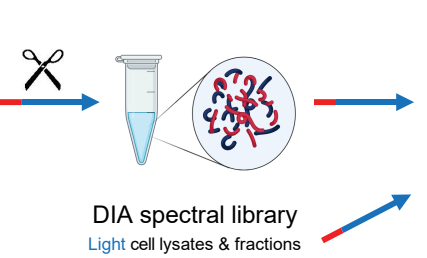

ADLNNENLEALEK

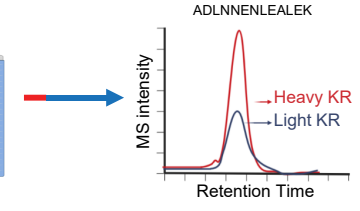

Absolute quantification

SILP-based concentrations for 16 anchor proteins

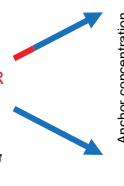

Label-free concentrations for $\sim 1,043$ proteins 



$\begin{array}{llll}\text { Flux Enzyme } & k_{\text {app }} & \begin{array}{c}\text { GPR } \\ \text { CAETHG_RS. }\end{array}\end{array}$

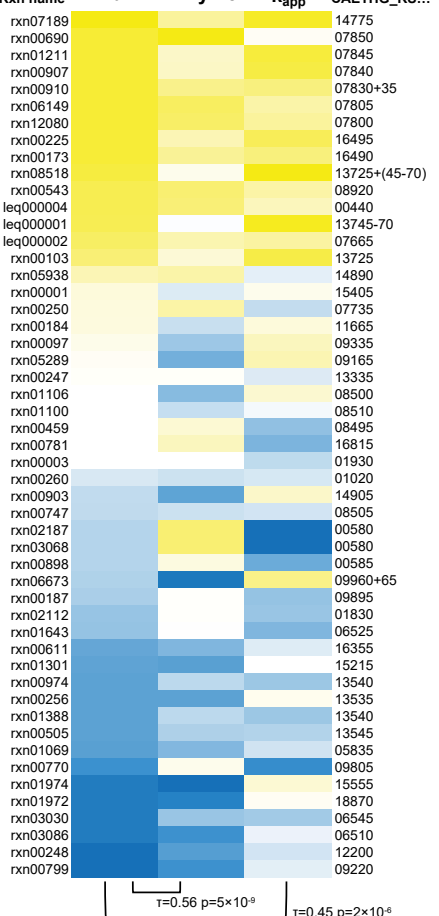

rxn00910

rxn06149

rxn08518

eq000001

q00012

rxn05938

rxn0000

rxn00184

rxn00097

rxn00247

rxn01100

rxn01100

rxn00003

rxn0090

rxn02187

rxn06673

זतn00187

rxn01643

rxn0061

rxn0130

rxn00256

rxn0138

$\times \times n 0106$

rxn01974

rxn01972

rxn03086

rxn0024

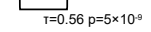

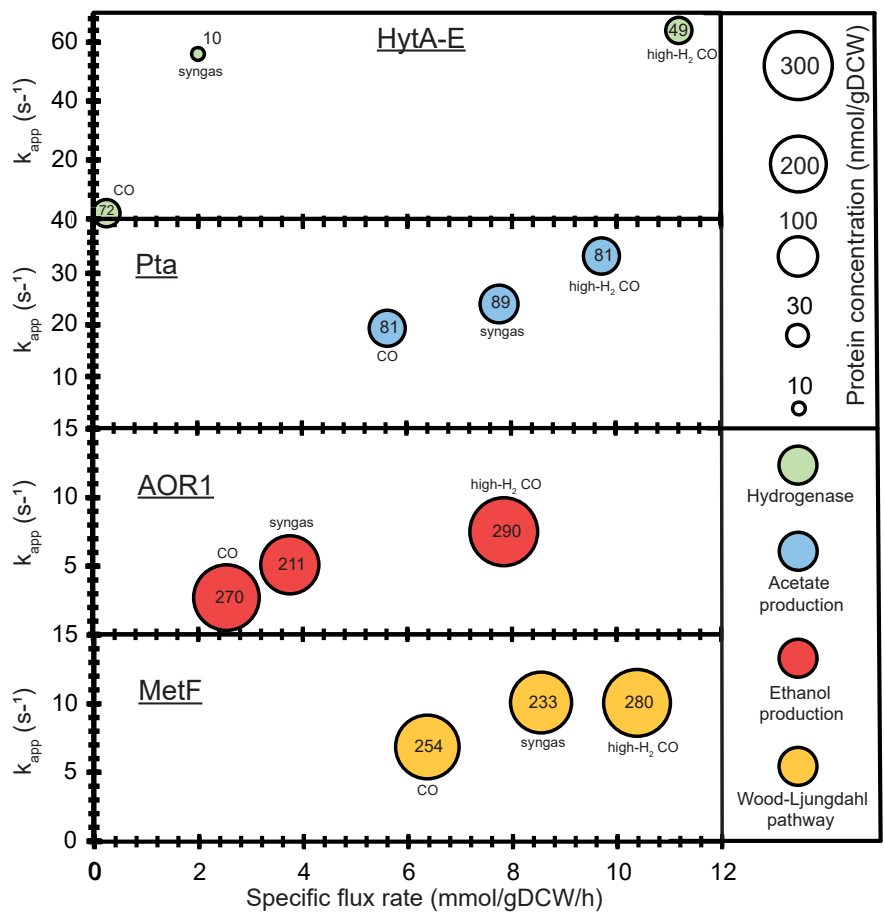

